\title{
La voix et les techniques vocales chez les Arabes
}

\section{Amnon Shiloah}

\section{OpenEdition}

\section{Journals}

Édition électronique

URL : http://journals.openedition.org/ethnomusicologie/1577

ISSN : 2235-7688

Éditeur

ADEM - Ateliers d'ethnomusicologie

Édition imprimée

Date de publication : 1 octobre 1991

Pagination : 85-101

ISBN : 978-2-8257-0437-8

ISSN : $1662-372 X$

\section{Référence électronique}

Amnon Shiloah, "La voix et les techniques vocales chez les Arabes », Cahiers d'ethnomusicologie [En ligne], 4 | 1991, mis en ligne le 01 janvier 2012, consulté le 30 avril 2019. URL : http:// journals.openedition.org/ethnomusicologie/1577 


\title{
LA VOIX ET LES TECHNIQUES VOCALES CHEZ LES ARABES
}

\author{
Amnon Shiloah
}

\section{La voix, reflet de l'âme}

«L'homme a un penchant naturel pour la voix» écrit le grand maître du soufisme Majd al-dīn al-Ghazālī (m. 1121), ajoutant dans le même ouvrage: «La voix du chanteur est un indice de la vie divine qui émane des profondeurs du mystère ». Nous pensons que les deux éléments de cet énoncé se trouvent dans un rapport de cause à effet. L'homme a un penchant naturel pour la voix parce qu'elle reflète les mystères de l'âme humaine qui, elle-même, représente les choses divines auxquelles elle appartient ${ }^{2}$. D'où cette autre idée courante qui compare la voix au souffle de la vie. La voix met donc l'homme en vibration avec le divin et l'univers et, comme moyen de communication sonore et verbal, lui permet d'exprimer, à travers une riche palette de timbres, les divers états de son âme et les subtilités de son être. A l'intervention de la parole, elle superpose avec ses modulations des surcharges affectives et psychiques au message purement linguistique.

L'importance de la voix et de ses vertus expressives est un sujet de prédilection de la littérature musicale arabe. Les observations et analyses qui lui ont été consacrées embrassent tous les phénomènes qui s'y rattachent, à savoir de l'émission de sons non articulés au chant sophistiqué, en passant par le parler ordinaire. Cette vue globalisante est soulignée par l'usage du même mot sawt pour désigner le bruit, le son, la voix, le phonème (en linguistique) et, par extension, un chant donné. Dans la gamme des produits de la voix, le chant alliant l'expression poétique et musicale se trouve placé au sommet de la hiérarchie et devient dans le domaine de l'art le symbole de la musique parfaite. Par conséquent, la musique savante reçoit la désignation de ghinā' (chant), réservant le terme emprunté au Grecs, $m \bar{u} s i \bar{q} \bar{l}$, essentiellement à la science musicale, qui était elle-même considérée comme un apport étranger ${ }^{3}$.

Majd al-dīn, grande autorité soufie, est le frère du célèbre penseur et théologien abū Hāmid al-Ghazāli (m. 1111). Les paroles citées ont été glanées dans son ouvrage Bawāriq al-ilmā‘... (cf. J. Robson 1938: 122, 158).

2 Défendue par les mystiques, cette doctrine réclame une existence divine préalable pour l'âme; elle est contestée par les théologiens de l'orthodoxie soutenant que l'âme naît avec le corps.

3 Dans les classifications des sciences médiévales, les Arabes distinguaient les sciences indigènes arabes (exégèse coranique, science de la Tradition, jurisprudence, théologie, grammaire, lexicographie, rhétorique et littérature) des sciences étrangères (philosophie, géométrie, astronomie, musique, médecine, magie et alchimie). 
En vertu de cette conception, la voix se trouve placée, dans la théorie, au centre de l'évolution musicale. Formulée par le célèbre philosophe et théoricien de la musique al-Fārābī (m. 950), cette théorie fait l'objet d'un exposé magistral figurant dans le premier discours de son traité monumental, Kitāb al-mūsīqī alkabìr "Grand livre sur la musique» ${ }^{4}$. Après une définition de la musique et de sa répartition en musique théorique et pratique, al-Fārābī présente une théorie de caractère rationnel sur l'origine de la musique et les diverses étapes de son évolution. Au premier stade de cette évolution, l'homme et l'animal emploient de la même façon leur voix et des sons spéciaux pour exprimer leur joie et leur douleur. La grande coupure apparaît quand l'homme commence à recourir à la parole; il ne se fie plus alors à son instinct qui, auparavant, lui permettait d'émettre des sons inarticulés pour s'exprimer. Avec l'aide de la raison dont il est doué, il a pu observer et analyser les rapports qui existent entre les sons spéciaux et les états d'âme, l'amenant à distinguer trois espèces de modulations: «Celles qui procurent du plaisir; celles qui provoquent des passions et celles qui donnent plus de portée aux paroles ». La dernière catégorie le pousse à une réflexion encore plus subtile, à savoir que la conjugaison des expressions poétique et musicale est de nature à rehausser l'art musical à son plus haut degré. Quant à l'instrument de musique, il n'apparaît, selon al-Fārābī, qu'au terme de la dite évolution; et même alors, en qualité d'accompagnateur seulement: «Les notes engendrées par tous les instruments sont de qualité inférieure, si on les compare à celles de la voix. Elles ne peuvent donc servir qu'à enrichir la sonorité du chant, à l'amplifier, à l'embellir, à l'accompagner... »

Ce bref résumé de la théorie d'al-Fārābī dégage et souligne la prédominance du concept de ghina $\bar{a}$ (chant) comme symbole de la musique la plus parfaite: la musique savante. Le ghinā' exprime donc l'union idéale des moyens de l'expression poétique et de l'expression musicale, qui est contrôlée et définie par des normes déterminées. Il appartient à l'artiste digne de ce nom de retrouver l'équilibre souhaitable entre les deux expressions, entre les idées et la forme.

Le concept de ghinā' que nous venons d'évoquer est exclusivement réservé à la musique savante; il ne s'applique donc pas aux genres religieux et populaires où l'idée de la prééminence du texte est exprimée par l'emploi du verbe «dire» à la place de «chanter». L'historien philosophe et sociologue arabe Ibn Khaldūn (m. 1406) va jusqu'à placer une limite nette entre deux formes d'expression $^{5}$. La première est qualifiée par lui de simple et instinctive; elle est à la portée de tous ceux qui possèdent un talent naturel mais sans aucune éducation musicale. L'exemple avancé pour illustrer cette catégorie est celui des lecteurs

\footnotetext{
Ce traité a été traduit en français par R. d'Erlanger et fait partie des volumes 1 et 2 de son ouvrage La musique arabe (1930-1959). Le texte arabe a été édité par Ghațas et Hefni (1967).

5 Cette théorie figure dans le chapitre sur la musique de son Muqaddima («Prolégomènes au métier de l'historien»). Cette œuvre est présentée comme une encyclopédie synthétique des connaissances méthodologiques et culturelles nécessaires à l'historien pour qu'il puisse faire vraiment œuvre scientifique. Il en existe une traduction française par de Slane (1863-68) et une en anglais par F. Rosenthal (1958).
} 
du Coran qui arrivent «à moduler leur voix comme si elle était une flûte; ils provoquent ainsi de l'émotion à travers l'harmonie qui résulte de leur mode d'expression». La deuxième forme d'expression se distingue par sa sophistication, qui est déterminée par les règles bien définies de l'art. Celles-ci exigent du musicien, en plus du talent naturel, des connaissances théoriques et une bonne maîtrise de tout ce qui concerne les fondements de l'art.

\section{Les divergences idéologiques}

Pour compléter ce tour d'horizon rapide, il convient de mentionner quelques divergences d'opinions qui opposaient les partisans du ghinā' à ses adversaires, qui appartiennent surtout à la classe des juristes et théologiens. Ces derniers s'attaquaient essentiellement au caractère distrayant et frivole du ghina $\bar{a}$, ainsi qu'à son effet subversif sur l'âme des croyants. La doctrine du formidable effet de la musique vanté dans la littérature fait référence à un autre concept prédominant, le tarab. A l'origine, le tarab signifie «s'extasier» devant la musique et la poésie quand celle-ci est récitée; dans son sens large, il assigne toute une gamme de réactions émotives allant de la douce délectation jusqu'à l'extase et même à un choc violent susceptible de causer la mort de l'auditeur ( $c f$. Shiloah 1972: 13). Considérant cette sensibilité devant le charme de la musique comme incompatible avec la foi, les opposants mettent tout en œuvre pour combattre ce qui envahissait alors bien des cœurs.

Les sources légales, théologiques et mystiques ${ }^{6}$, dans le cadre des débats relatifs à la musique, emploient le terme samā' (litt. «audition»). Le samā', dans son sens le plus large, comprend les phénomènes sonores et gestuels, à savoir la récitation poétique à caractère mélodieux, la cantillation du Coran et l'appel à la prière, les hymnes métriques et, dans le contexte mystique de certaines confréries, l'intervention instrumentale et la danse. Pour contrecarrer les critiques acerbes formulées à l'égard de ces mystiques épris de musique, leurs défenseurs soutiennent que leur manière n'a rien à voir avec le ghin $\bar{a}$ ' et le tarab et qu'elle ne doit aucunement être assimilée à la nature du lahw (distraction). Toutefois, parmi les mystiques qui assignaient à la voix et à la musique une origine divine, se trouvaient à l'occasion des attitudes non conformistes qui ouvraient la porte à l'infiltration de la musique savante dans le domaine du sacré ${ }^{7}$. Inutile d'insister sur le fait que de telles attitudes accroissaient l'intransigeance des docteurs et des théologiens extrémistes.

Le lecteur intéressé en trouvera un bon nombre de références dans Shiloah (1979).

7 Ceci est particulièrement vrai dans les pratiques de la confrérie des Mevlevis, connue en Europe sous le nom des derviches tourneurs. Elle fut fondée par le poète et philosophe Djalāl al-dīn al-Rūmī (m. 1273). Leur cérémonie appelée Mukabele est étudiée dans Molé (1963) et Ritter (1955). Pour une vision globale de la musique et de l'extase chez les mystiques, voir During (1988). 
Pourtant il y a une forme d'expression qui a été acceptée par presque tout le monde: celle où les idées et leur contenu prédominent sur le musical, c'est-àdire la cantillation ou la psalmodie, qui embellit la lecture des textes coraniques et l'appel à la prière. Celle-ci serait, dans le même ordre d'idée, caractéristique de la récitation mélodieuse de la poésie classique arabe avant l'avènement de l'Islam. En effet, cette poésie était orale et prenait toute son envergure dans la récitation publique. Était-elle vraiment chantée? Elle le fut assurément en partie; le revêtement mélodique devait être rudimentaire, ce qui la rapprochait des caractères essentiels de la cantillation. Mais même récitée, comme on peut encore le constater de nos jours, ce mode d'expression comporte un caractère chantant prononcé qui semble être spontané dans la langue arabe aux dires de l'éminent orientaliste Louis Massignon. Dans un bref article intitulé «Voyelles sémitiques et sémantique musicale» (1961: I, 79-83), celuici explique comment les voyelles, qui sont fondamentalement distinctes des consonnes, personnalisent et vivifient le squelette muet de ces dernières. La triple vocalisation ou, $a, i$ est selon l'auteur, «la base de la grammaire arabe et la base de la sémantique musicale sémitique». Il dit plus loin: «La psalmodie, cet infra-chant qui, ailleurs, n'est pas lié à un phénomène grammatical déterminé, naît en sémitique avec la nunnation arabe. La nunnation a déterminé la psalmodie nasalisée, sorte d'accentuation émotive provoquant l'attention». Cet esprit qui, comme on le verra, a conféré une ampleur particulière à la lecture solennelle du Coran, animait aussi la récitation mélodieuse de la poésie anté-islamique; la métrique quantitative caractérisant cette poésie ne semble pas avoir entravé l'expression musicale.

Dans une étude récente sur l'expression poétique d'Homère, Haiim Rosen, cherchant à mieux apprécier l'art homérique en tant que poésie orale, souligne que dans cette poésie orale basée sur une métrique quantitative, il y aurait eu des compromis dans les accentuations dues à la mise en relief phonique, à l'emphase et à l'intensité. Il conclut en disant: "C'est uniquement en saisissant correctement et de manière adéquate les moyens de l'expression poétique qu'on aboutit à apprécier avec justesse l'art du poète et surtout l'équilibre qui règne entre les deux fondements de la poésie: d'un côté les idées, les valeurs émotionnelles, les images et les associations suscitées, de l'autre la musique et le rythme» $(1989: 274)$.

\section{Définitions et terminologie}

L'affinité qui, dans l'expression verbale rehaussée, allie la rhétorique, la psalmodie et le chant, se dégage dans la terminologie, notamment dans les sens dérivés de la racine gny (chanter, et aussi prolonger la voix dans la psalmodie, enrichir, s'enrichir et se passer de), et de la racine lḥn dont dérive laḥn (mélodie, rythme et mode), signifie aussi psalmodier de façon agréable à l'oreille de l'auditeur, élucider une parole et en faire ressortir le sens, voire même com- 


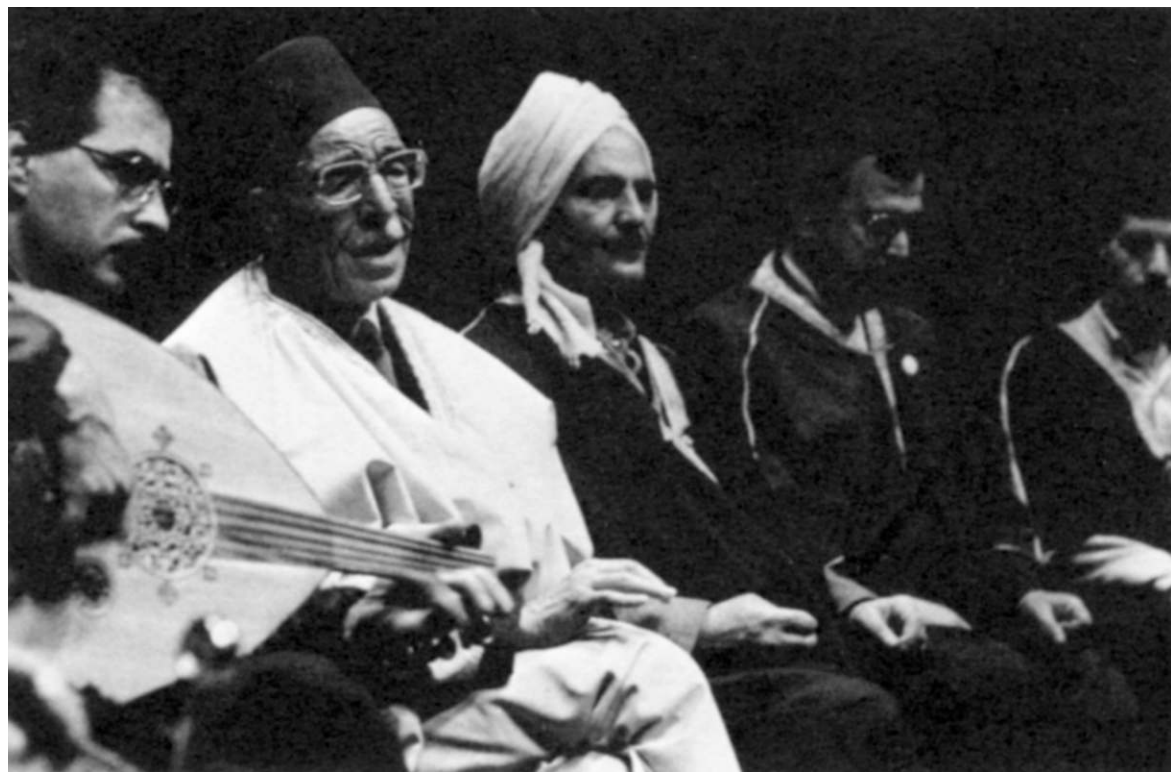

Wasla d'Alep, Syrie: Sabri Mudallal et son ensemble. Photo: Alan Humerose, 1988.

mettre des fautes dans la lecture ${ }^{8}$. Dans un traité relativement tardif $\left(\mathrm{XV}^{\mathrm{e}} \mathrm{s}\right.$.), Le commentaire anonyme sur kitāb al-adwār (Livre des cycles) du célèbre théoricien Șafī al-dīn (m. 1294), l'auteur anonyme écrit dans sa définition de la musique: «Le mot musique (mūsìqi) est grec; il a pour sens les mélodies (alhāan). Quant au terme laḥn (sg. de alhạnn), la lexicologie arabe lui donne le sens de lecture qui donne des émotions. Ainsi, quand quelqu'un lit sous l'empire d'une émotion, on dit qu'un tel laḥhana, ou encore gharrada dans sa lecture. [...] Le taghrïd (nom d'action de gharrada) est l'émotion dans le son » (Erlanger 1930-59, III : 190). Un autre auteur, encore plus tardif, l'encyclopédiste Hadjdjī Khalīfa (m. 1657), se voit amené à inclure dans sa définition de la musique la rhétorique, ou l'art des orateurs, ainsi que la cantillation (Shiloah 1979).

Ce trait d'union terminologique n'est pas une pure spéculation lexicographique; il est essentiel pour une approche conceptuelle. Il se manifeste dans les premiers siècles de l'Islam dans les rapports étroits entre rhétorique, expression poétique, prosodie, cantillation et musique savante. Dans tout cela la transmission verbale, précise et soutenue par la richesse des émissions vocales,

Ce dernier sens est probablement à l'origine de la forme de poésie dialectale maghrébine, le malhūn. Dans la nouvelle édition de l'Encyclopédie de l'Islam il est dit que le terme vient de «laḥana pris dans le sens de "s'écarter de la norme linguistique", en l'occurence de l'arabe littéraire». 
joue le rôle de pivot. La survie de cette approche, plusieurs siècles après l'avènement de l'Islam, prouve sa validité.

A l'époque de la formation de la nouvelle civilisation, on voit apparaître chez les conquérants musulmans un sentiment d'attachement au passé, à ce qu'on porte en soi et qu'on veut préserver; ce sentiment serait à l'origine du processus de l'arabisation comme objet de l'unification culturelle. Le souci de pureté linguistique qui prédomine dans ce processus conduit à l'étroite dépendance entre la lecture du Coran, l'interprétation du chant d'art et les origines de la grammaire arabe.

\section{Concepts esthétiques}

Un thème favori qu'on rencontre fréquemment dans les ouvrages littéraires du passé est celui d'énoncer les mérites et les caractères distinctifs des divers peuples connus en se référant à leur musique. Un des plus célèbres littérateurs, l'auteur prolifique al-Djāhịiz (m. 868), écrit dans l'un de ses nombreux passages sur la musique: «Les Persans faisaient du chant une branche des belles-lettres $(a d a b)$ et les Grecs l'assimilaient à la philosophie, quant aux Arabes, ils distinguaient poésie, rhétorique et éloquence» (Shiloah 1979: 91-2). Cette constatation souligne indirectement l'apport arabe à la nouvelle musique, dans laquelle l'expression poétique prête son concours à l'expression musicale. Cette façon de voir figure avec plus de précision sept siècles plus tard dans le commentaire anonyme du Livre des cycles de Șafī al-dīn. Dans sa définition de la musique, le commentateur anonyme écrit: «Le but de l'art musical est d'aider à atteindre celui de l'art poétique». Pour bien préciser qu'il ne s'agit pas là simplement d'une prolongation du sens du texte, l'auteur propose une clarification vers la fin du traité. Sous le titre «De la composition des mélodies» (Erlanger 1930-59, III : 519 et 539), il inclut un long exposé sur les principes de base de tous les modes d'intervention vocale. Bien que certaines idées ne fassent que résumer des propos tenus par d'autres auteurs, l'ensemble de l'exposé, avec son enchâ̂nement systématique, est tout à fait remarquable. L'auteur distingue d'abord deux espèces de musique vocale: l'une est à rythme et forme musicale libres; elle concerne la cantillation, les adresses oratoires et la déclamation des poésies. L'autre est conçue dans une forme mélodique et rythmique déterminée. Une deuxième distinction générale fait appel à la dichotomie quantité-qualité. Par quantité, il désigne les valeurs mesurables de l'acuité et de la gravité des notes qui sont d'ailleurs communes à l'instrument et à la voix. La voix, de son côté, se distingue par des qualités propres aux notes qu'elle émet. Là, l'auteur s'emploie à énumérer un grand nombre de timbres qui sont les produits des émissions vocales et des sons spéciaux qui dénotent des états d'âme. Parmi ces qualités figurent celles qui déterminent les phonèmes. Comme ceux-ci sont produits par des parties spéciales des organes vocaux, il s'ensuit une présentation détaillée de phonétique musicale. Enfin, l'auteur passe aux notions de base de 
la prosodie et de la métrique, auxquelles d'ailleurs recourent tous les théoriciens de la musique pour expliquer les fondements de la composition et des formules rythmiques. Et pour conclure, l'auteur dit: «L'art musical consiste à adapter des paroles aux mélodies et des mélodies aux paroles de telle façon que les phonèmes qui composent les paroles deviennent des qualités distinctives par rapport aux notes des mélodies ».

En suivant cette même démarche, nous pouvons admettre que le chant d'art, en dépit de son éloignement de la zone où le musical se trouve assujetti au verbe et à ses inflexions, demeure toujours sous son égide idéale et continue à manifester son attachement aux conceptions de base des formes et principes de la récitation mélodieuse et de la cantillation.

\section{La cantillation}

La cantillation, dont le but principal est de régler la restitution exacte des textes sacrés, a fait l'objet d'études minutieuses dès le début de l'Islam. Vu son importance capitale, sa systématisation prend rapidement une ampleur considérable englobant l'analyse détaillée des phénomènes phonétiques, des timbres et des types de voix désirables. La codification de ses normes visait avant tout à assurer la bonne diction et à prévenir les déviations dangereuses dans la récitation. Toutefois il ne serait pas hasardeux d'admettre que ses normes exerçaient une influence remarquable sur la musique savante, surtout si on pense au processus d'arabisation mentionné plus haut.

Comme appui à l'étude de la cantillation, les auteurs recourent au verset coranique: «Psalmodie le Coran avec soin» (S. LXXXIII, 4). Cette prescription conduisit à l'établissement du tadjwìd comme branche des sciences coraniques. Le tadjwìd (la parure de la récitation) est un système consacré à la voix et à ses émissions, ainsi qu'à l'art de psalmodier selon les règles et les lois de la phonétique. Quant au revêtement mélodique, suivant les sources, il aurait été inspiré des airs simples qui accompagnaient les chants de chameliers, le hid $\bar{a}$ ' et le rakbānì. Le hid $\bar{a}$ ' est considéré comme la première forme du chant arabe préislamique (Farmer 1929). Une tradition (hadith) ${ }^{9}$ rapporte à cet effet que le Prophète recommanda de lire le Coran avec des mélodies arabes de la région de Najd en Arabie et non pas avec celles des mécréants, des Juifs et des Chrétiens. L'auteur de l'importante Encyclopédie des sciences coraniques, al-Suyūṭi (m. 911), mentionne une autre tradition qui indique exactement le texte du chant dont l'air fut emprunté pour la psalmodie. Ce même auteur cite une autre tradition ayant trait à une caractéristique majeure de la récitation:

Hadìth (récit, propos) est employé, selon l'Encyclopédie de l'Islam, pour désigner la Tradition rapportant les actes et les paroles du Prophète, ou son approbation tacite de paroles ou d'actes effectués en sa présence. 
la pause. Abu'l Faḍl al-Rāzī fut, dit-il, le premier à avoir attiré l'attention sur la question de la pause, tout en s'inspirant des lois de la prosodie. Enfin, il convient de signaler que le genre hida , le chant du chamelier qui aurait inspiré la psalmodie à ses débuts, était d'un caractère plaintif et que, selon les témoignages anciens, il se confondait avec la lamentation. Or, un caractère plaintif était recommandé pour la psalmodie.

Nous supposons que cette espèce de réévaluation était guidée par des besoins culturels innés, mais l'on est en bon droit de se demander s'il n'y avait pas aussi un aspect de compromis avec les coutumes que l'on transformait pour les besoins de la cause. Le témoignage suivant d'Ibn al-'Arabī (m. 844-6) semble confirmer cette possibilité: «Le Prophète voulut que le Coran devint leur passion exclusive en remplacement du rakbānì (un genre de chants de chameliers) qu'ils avaient coutume de chanter ${ }^{10}$. Quoiqu'il en soit, la reconversion ne fait en fin de compte qu'accentuer les valeurs intrinsèques et encourager leur survie.

\section{Échelle de timbres et de qualités vocaliques}

L'art du tadjwìd comprend deux constituants: le premier, d'ordre phonétique, est dénommé taḥì q, c'est-à-dire l'établissement avec précision de chaque consonne en lui donnant sa pleine valeur et tout ce qu'elle comporte pour être bien prononcée; le second est le tartìl qui concerne les règles de la récitation, son mouvement, la force et l'emphase marquées par la voix et les pauses. Deux vers tirés d'un des nombreux poèmes didactiques illustrent ces conditions:

Une consonne a une valeur propre: respecte sa mesure et ne détériore pas sa nature.

Ne pense pas que le tadjwid est une prolongation sans limites ou bien celle appliquée à ce qui ne se prête pas à être étendu.

Quant à la partie phonétique de la science du tadjwìd, elle procède d'une analyse minutieuse de la formation des diverses consonnes dans les organes de l'appareil phonatoire (poumons, pharynx, fosses nasales, luette, langue, lèvres) afin de dégager ainsi les qualités et les nuances vocales correspondantes que le lecteur avisé doit s'employer à produire avec précision. Signalons à cet effet que les vingt-quatre lettres de l'alphabet arabe sont réparties en classes phonétiques, à savoir les gutturales, les labiales, les nasales, etc. A cela s'ajoutent deux facteurs: le degré d'aperture de la bouche qui permet de distinguer parmi les consonnes occlusives, spirantes, liquides, etc., et le mode d'articulation

10 Il s'agit d'une tradition figurant dans le recueil de Bukharī, qui fait autorité; elle est citée dans Talbi (1958), où celui-ci fait grand cas du recours à la musique savante dans la récitation du Livre saint. 
qui permet de classer les consonnes en sonores, emphatiques, sourdes, géminées, etc. Chaque son acquiert ainsi une qualité propre dénotant d'une part le caractère technique de son émission et, d'autre part, les nuances phonétiques influençant l'impression auditive lors de la prononciation. Cette dernière doit être assurée sans difficulté ni exagération. Elle est qualifiée soit de tafkhìm, prononciation pleine, grasse ou grave; soit de tarqīq, prononciation fine et aiguë. D'autres qualités se nomment: tafashshī, étalement du souffle dans l'articulation du sh; idghām, assimilation, et ghunna, nasalisation, qui joue un grand rôle dans la lecture. Ainsi, tous les phénomènes phonétiques sont désignés par des termes appropriés et sont scrupuleusement analysés dans les traités du tadjwìd, souvent aussi en rapport avec le chant d'art.

Ainsi qu'il l'a été mentionné auparavant, les consonnes arabes sont imprononçables, inertes; ce sont les voyelles, considérées comme mouvement (harakah), qui les vivifient, les personnalisent, d'où l'importance capitale de leur présence conjointe à celle des consonnes dans l'émission du son. La science du tadjwìd considère en l'occurence le traitement et la nature des trois voyelles fondamentales sous différents aspects, notamment celui de l'imālah, inclinaison de la voyelle a vers le son $i$, ou encore celui de la durée de l'émission. Or, la durée des voyelles est conditionnée par les consonnes qu'elles mettent en mouvement, la fonction du mot dans la phrase et sa place dans le verset.

Le tartìl, qui est le second objectif du tadjwìd suivant la définition généralement acceptée, touche à la restitution globale du texte sous une forme compréhensible et susceptible de toucher les fidèles. L'agencement du texte avec les pauses et les arrêts adéquats, ou l'alternance correcte de l'écoulement rythmique et des repos, assurée par un contrôle rigoureux du souffle, est considérée comme la condition fondamentale de la cantillation. En effet, c'est d'elle que dépend la transmission du message contenu dans le texte, car s'arrêter à un endroit inadéquat de la phrase risque de conduire à une interprétation entachée d'hérésie. Ainsi, en observant les règles établies, le lecteur devient en quelque sorte l'interprète et le porte-parole de la version officielle. Les cinq règles essentielles de la récitation, qui soulignent aussi sa dimension musicale, sont: l'istirsāl, la prolongation du son sans laisser tomber la voix; le tarkhìm, l'adoucissement du son sans perdre l'intonation; le tafkhim, l'amplification du son; le taqdìr al-anfās, le contrôle de la respiration; et le tadjrìd, la transition maîtrisée d'un son fort à un son faible et vice-versa.

Enfin, les auteurs du tadjwìd incluent dans leur exploration un examen approfondi sur les qualités des voix recommandées pour la cantillation. Parmi plus de trente variétés de voix répertoriées, seules onze sont retenues, car les autres ne sont pas jugées compatibles avec la cantillation. Il convient de signaler que les types de voix recommandés pour la lecture du Coran, ainsi que leurs désignations respectives font partie de la nomenclature complète que présentent et analysent les théoriciens de la musique savante. Ceci est vrai dans une large mesure pour une grande partie des autres caractéristiques du tadjwìd. Nous reviendrons sur la question des types de voix en parlant de la musique savante. 


\section{La belle voix ${ }^{11}$}

La notion de belle voix et sa définition apparaissent fréquemment dans la littérature relative tant à la cantillation qu'à la musique savante. Son évocation dans le contexte religieux est souvent accompagné de modèles dénotant parfois un caractère apologétique. Ainsi, pour vanter le grand pouvoir expressif de la belle voix, l'on cite l'exemple de l'ange Serafil ${ }^{12}$ et celui du roi David. Une tradition répétée assure que l'ange Serafil a la plus belle voix que Dieu ait créée. Quand il chante, tous les habitants du Ciel arrêtent leur prière et leurs louanges, les arbres bourgeonnent, les oiseaux et les Houris (femmes paradisiaques qui se distinguent par leur voix superbe) ${ }^{14}$ mûs par l'inspiration, se mettent à entonner des hymnes qui font les délices de tous ceux qui s'étaient abstenus du plaisir musical dans ce bas monde. Quant à David, le pouvoir qu'on lui attribuait de psalmodier avec la plus belle des voix fut à l'origine d'une tradition qui raconte que Mahomet, pour exprimer son exaltation devant la belle voix d'abū Mūsā al-Ash'arī, dit: «Tu as reçu un des mizmār qui appartenaient à David ». ${ }^{15}$ Mizmār signifie ordinairement un instrument à anche, mais, dans ce cas, ce terme correspondrait à mon avis à mazmūr (mizmor en hébreu) qui veut dire psaume, impliquant la belle psalmodie de David. Par ailleurs, on attribue à la belle voix de David un pouvoir affectif extraordinaire, un charme irrésistible sur les oiseaux, les fauves et les êtres surnaturels, au point que la proximité de cette psalmodie fait tomber de nombreuses victimes. Enfin, il est dit que sa voix retentira le jour de la résurrection.

L'insistance sur cette notion de belle voix dans les sources les plus diverses nous conduit à la question de savoir en quoi consiste la beauté de la voix; y a-t-il des critères précis qui la distinguent? Disons-le tout de suite, dans le contexte religieux, la notion de beauté n'est jamais assimilée à une quelconque catégorie esthétique; on la considère uniquement sous l'angle de son expressivité éthique, destinée à donner l'ampleur nécessaire à la récitation des textes sacrés, et comme moyen de rendre le message plus touchant. Il n'en va pas de même dans le contexte de la musique savante où la belle voix est appelée à séduire, à charmer.

Dans un traité d'al-Djāhị sur les esclaves-chanteuses, qui est la première œuvre en prose à traiter de l'amour sous la forme d'un essai, il y a trois sens

\footnotetext{
11 L'adjectif al-hasan signifie beau, bon et bien.

12 Serafil, ou Israfil, est l'ange de la résurrection, dont l'attribut est la trompette.

13 David fait l'objet d'un certain nombre de passages coraniques où ses qualités sont vantées. Les commentateurs du Coran et les historiens des prophètes le dépeignent comme prophète et insistent sur son habileté à psalmodier.

14 Les Houris sont, d'après le Coran: «aux regards modestes, aux yeux grands et beaux que ni homme, ni démon n'aura touchées avant eux» (LV 72-74).

15 Abū Mūsa al-Ash'arī était compagnon du Prophète et l'un de ses principaux lieutenants.
} 


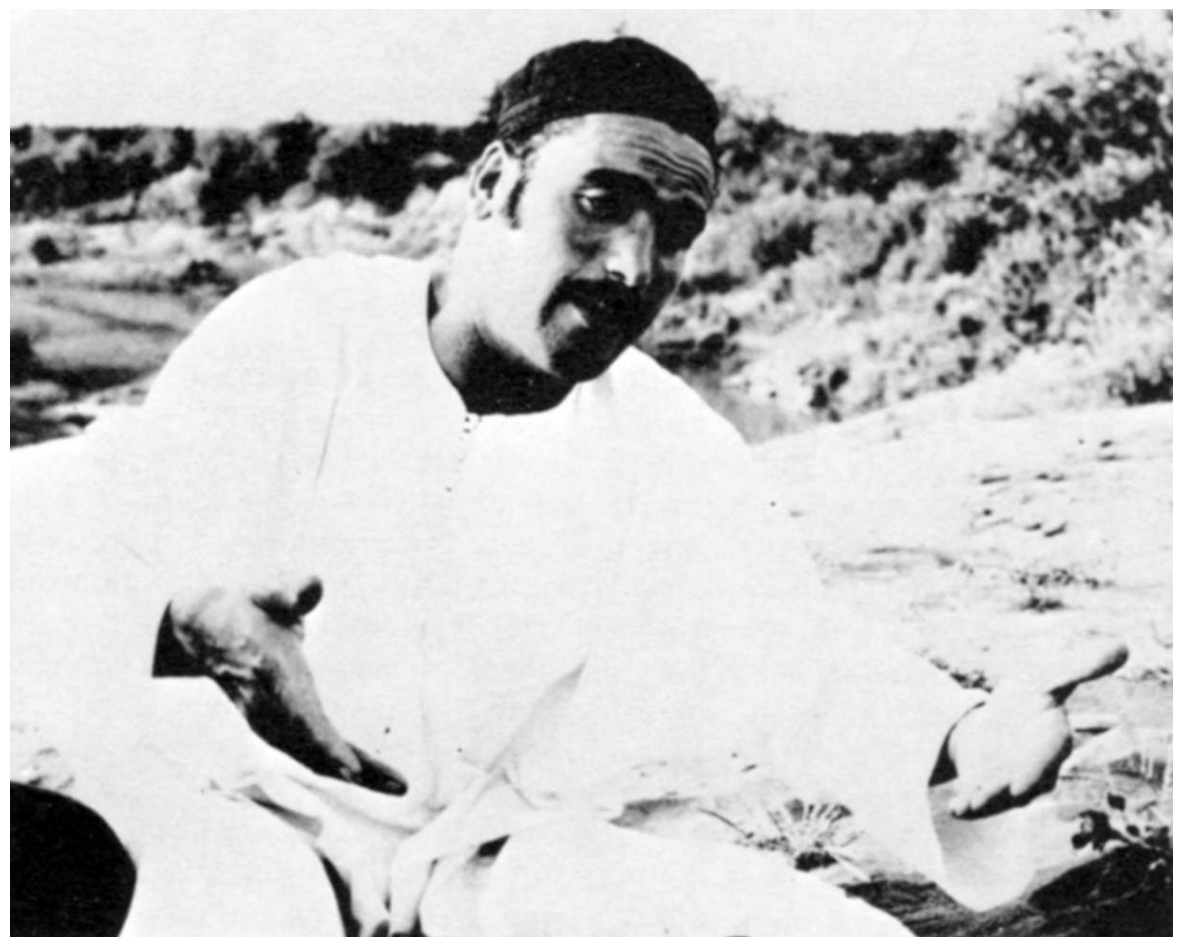

Ahmed Slassi, chanteur populaire. Ksar-el-Kebir, Maroc, 1970. Photo : Jean-Claude Brutsch, 1970.

qui sont associés comme le dit R. Sicard (1987) «pour la vue, la contemplation d'une belle et appétissante esclave, car l'habileté [professionnelle] et la beauté se rencontrent rarement pour le plaisir des amateurs; pour l'ouïe... la joie procurée par la musique, pour le toucher, le désir sexuel et l'envie du $b \bar{a} h$ (coït)...». Des variations sur cette espèce d'association sont multiples dans les écrits appropriés. Six siècles après al-Djāḥiz, le poète ottoman Muḥyī al-dīn ibn al Khațīb (m. 1533) écrit que le chanteur doit remplir trois conditions pour être digne de sa profession: avoir un beau visage, une taille élancée et une expression douce. Et il ajoute que, si le chanteur est laid, il fera mieux de se cacher en chantant pour que sa laideur ne fasse pas ombrage aux délices de sa voix. Et pour conclure, il paraphrase al-Djāhịiz disant: «Quelle différence y a-t-il entre chant émis par une bouche qui te donne envie de l'embrasser et un chant entonné par une bouche qui t'inspire de détourner le regard?» ${ }^{16}$.

16 Ce passage figure dans la rawḍa 31 de l'Anthologie littéraire intitulée Raw ḍa al-akhyār.... 


\section{Classification et classement des voix}

En traitant de la voix et de ses qualités, on retrouve chez les théoriciens et musicographes le même souci de minutie et de recherche de nuances. Là aussi, on associe les phénomènes sonores à la phonétique, à la grammaire et aux diverses particularités de la langue arabe.

Pour illustrer notre propos, voici un exemple qui se distingue par des traits particuliers. Il s'agit de La perfection des connaissances musicales dont l'auteur, al-Hasan al-Kātib, semble avoir vécu au XI siècle (cf. Shiloah 1972). Ce traité appartient à une catégorie spéciale d'écrits; il combine, selon la définition de son auteur, la théorie et la pratique musicales de l'époque. Il touche en effet à des problèmes concrets qui sont remarquablement analysés par un observateur qui semble avoir été un éducateur. Un coup d'œil rapide sur les quarante-trois chapitres composant ce traité nous montre d'emblée les affinités entre musique, phonétique, rhétorique, poésie et prosodie, sur lesquelles nous avons insisté dans cet article. Parmi les sujets traités séparément, fixons notre attention sur deux chapitres: le XXIII, qui traite des articulations et des données phonétiques, et le XXXV, qui expose les caractéristiques des voix.

Le premier suit de très près l'enseignement du tadjwìd énumérant quarante-sept sons préférentiels désignés par des dénominations propres. Une partie d'entre eux couvrent des phénomènes phonétiques, des articulations et des nuances vocaliques, les autres se réfèrent à des notions de hauteur, d'intensité, de puissance et de couleurs, leur assignant des expressions particulières. La parenté avec l'art du tadjwìd est surtout évidente dans la partie qui concerne la formation des lettres, leur articulation et leur prononciation exacte; nous y retrouvons le même développement, la même terminologie. Par ailleurs, une bonne partie de la nomenclature a trait à des faits d'ordre psychophysiologique correspondant à des moyens susceptibles de créer des émotions et de doter l'interprètation musicale d'un surplus d'expressivité. Les termes qui se rapportent à la hauteur et à la durée sont par exemple: le sadjāh qui désigne le son grave d'une octave et le șiyāh , sa réplique à l'aigu. A cela, il convient d'ajouter l'important procédé d'octaviation, considéré comme un facteur d'embellissement par excellence; le choix de l'ambitus en fonction des possibilités de la voix du chanteur, et le tadrìdj (gradation), l'évolution graduelle du grave à l'aigu et vice versa en observant l'homogénéité de timbre. La durée est impliquée dans un terme comme al-tamatți, action de prolonger un son avec le souffle aussi longtemps qu'il est possible.

Quant aux sons expressifs spéciaux, il nous semble bon d'en mentionner une douzaine, vu leur importance dans le système. Les termes seront suivis d'un bref commentaire. Sarkhah, son aigu, tenu et unique; dadjarah, son d'angoisse; nazhah, soupir d'élan; istighäthah, cri plaintif qui fait penser à l'appel au secours; ta'awwuh, l'interjection «ah! » qui traduit un soupir d'angoisse; qahqahah, son rappelant l'éclat de rire; hazzah, tremblement; tanahhud, une ou plusieurs notes suivies d'un soupir manifeste à la manière d'un sanglot; sahîl, notes émises graduellement à la manière du hennissement d'un cheval; 
hamzah, son de poitrine (occlusion glottale); zakhmah, resserrement; takahhun, sons accompagnés d'un bourdonnement de gorge.

Cette riche palette de timbres, je dirais cette rhétorique sonore et musicale, est probablement à l'origine de la multiplicité de types de voix que les auteurs arabes distinguent dans leurs écrits. Parmi les quelque trente variétés de voix connues, seules onze ont été retenues comme souhaitables pour la psalmodie; elles font d'ailleurs toutes partie des qualités énumérées dans le chapitre XXXV d'al-Hasan al-Kātib. Il est intéressant de signaler à ce propos qu'une liste plus ou moins analogue à celle d'al-hasan figure chez un auteur égyptien du XX ${ }^{\mathrm{e}}$ siècle: Kamil al-Khulay (cf. Rouanet 1922). Est-il possible d'en conclure que la conception dont nous traitons aurait survécu aux vicissitudes du temps? Je pense qu'au début de notre siècle, ceci aurait pu être vrai.

Les qualifications des voix proposées par les deux auteurs précités sont: affligée ; vacillante ; ronde comme un bassin ; complaisante ; sonore/claire; puissante; rugueuse/dure; douce/lisse/légère; pure/homogène; excessive/surabondante; claquante; retentissante/métallique; criarde/perçante; tremblante; nasillarde; tendre/humide; étouffée; moelleuse; obscure/sombre; menue/ délicate; réverbérante; suffoquée; flasque/nonchalante; agitée/perturbée; brisée/saccadée; rauque.

Dans cette avalanche de qualificatifs propres à nous émerveiller, il n'est pas question de classement de voix par hauteur; la classification concerne exclusivement le timbre, la puissance, l'éclat, l'épaisseur et le volume. En examinant de près la nomenclature et les témoignages, on pourrait relever une préférence générale pour les voix aiguës affectées, légèrement rauques et nasillardes.

Cette richesse de nuances et de qualités vocales souligne aussi la faculté de la voix humaine d'aboutir dans sa haute subtilité au degré lui permettant de jouer le rôle de porteuse et génératrice d'émotions qui se précisent et s'intensifient avec le concours de la parole. Mais la voix, indépendamment du rôle important qui lui est assigné dans l'interprétation musicale pure, possède un caractère expressif en soi.

C'est ainsi que le théologien al-Ghazālī (m. 1111) explique l'hostilité des juristes à l'égard du chant de la femme, car, dit-il, l'interdiction n'a pas été motivée par la musique mais par la voix; en effet, la voix féminine est séduisante même en causant ${ }^{17}$.

17 Cet énoncé figure dans le chapitre sur les lois du samā' qui est le huitième livre du deuxième «quart» de son Ihȳa 'ulüm al-dīn («Revification des sciences de la religion»). A ce propos, il convient de signaler un approche semblable dans le Judaïsme, qui s'appuie sur le dicton talmudique Qol be-'ishshah 'erva : «la voix de la femme est impudicité» ou «femme chantant en public commet un acte impudique». 


\section{Le traitement de la voix}

Pour le bon maintien de sa voix, le chanteur est tenu d'en prendre soin quotidiennement. Cet aspect des choses fait l'objet d'un chapitre particulier du même al-Hasan. Il s'agit des recommandations pratiques concernant surtout le régime alimentaire à observer. A ce propos, notre auteur conseille l'absorption d'eau chaude ou d'huile d'amandes à jeun, ou quand on a faim. Il recommande le jus de légumes, le froment tendre broyé mélangé avec du beurre et du sucre, la canne à sucre grillée, le jus de violette dilué dans de l'eau chaude, l'œuf à la coque, la consommation de prunes et la succion de la plante djullāb (julep).

Par ailleurs, la fréquentation de l'étuve, les exercices soutenus ainsi que la récitation modulée, sont de nature à augmenter la beauté de la voix. De plus, il est conseillé au chanteur d'éviter le surmenage de sa voix, les longues veillées, les chants à danser qui imposent des contraintes à la voix, le vent frais et le froid, et enfin les aliments acides.

\section{La formation du chanteur et les développements de ses activités vocales}

Il est bien entendu que le postulant doit avoir au départ un talent musical naturel, une belle voix, un sens inné du rythme et une faculté d'assimilation rapide. Pour mettre à l'épreuve ces dispositions, le maître demande au postulant de chanter différents genres de musique en observant l'émission des lettres et l'articulation. Ensuite, il examine sa déclamation poétique et la puissance de sa voix. Si le postulant est admissible, son entraînement comportera l'amélioration des postures musculaires, le conditionnement de l'émission vocale et de l'ouverture buccale, les modalités respiratoires et articulatoires. Ce travail a pour but d'agir sur les timbres et la modification des couleurs vocaliques pour enfin aboutir à la bonne diction et à la maîtrise dans l'émission des sons expressifs. Au niveau de l'interprétation, le maître est censé choisir pour son élève des chants qui s'adaptent le mieux à sa voix et à ses capacités artistiques; un mauvais choix risque de nuire à la voix du débutant et à son potentiel artistique. Pour former son goût, l'élève doit écouter longuement les grands artistes et observer leurs manières.

Nous possédons un témoignage célèbre rapporté par l'historien de l'Espagne musulmane al-Maqqāri (m. 1632) ${ }^{18}$ qui décrit la méthode d'enseignement développée par l'illustre musicien Ziryāb ${ }^{19}$. Selon al-Maqqāri, «Quand

Al-Maqqārī écrivit une longue monographie sur l'Espagne musulmane et sur le polygraphe grenadin Lisān al-dīn ibn al-Khațīb sous le nom de Nafh al-țîb... C'est une immense compilation de renseignements historiques et littéraires (cf. Dozy et al., 1855-61: II, 83-91; Abbas 1968: III, 122-33). 
Zyryāb entreprenait la formation d'un élève, il l'invitait tout d'abord à s'asseoir sur un coussin rond connu sous le nom de miswara, puis, si celui-ci possédait une voix forte, il lui demandait de chanter à gorge déployée; si sa voix était faible, il lui prescrivait de serrer sa taille avec un turban, car ce moyen avait le don de renforcer la voix. En effet, la colonne d'air ne se disperse pas dans ce cas en s'acheminant vers l'ouverture buccale. Si l'élève avait le défaut de tenir ses molaires rapprochées et de ne pas pouvoir ouvrir la bouche, ou s'il avait l'habitude de serrer ses dents en parlant, il le soumettait à un exercice qui consistait à introduire dans sa bouche un morceau de bois, large de trois doigts, et à l'y laisser pendant quelques nuits jusqu'à ce que ses mâchoires fussent élargies. Par ailleurs, s'il voulait mettre à l'épreuve un postulant pour savoir s'il avait ou non un talent inné pour le chant, il l'invitait à crier de toutes ses forces ô hadjjjām (ô poseur de ventouses) ou à crier $A h$ ! en prolongeant sa voix. Si, en entonnant ces mots, sa voix était claire, retentissante, puissante et porteuse, sans être dépourvue de nasalité et sans souffrir d'étouffement ou de manque de souffle, il était certain que cet élève était qualifié et il acceptait de le former; sinon, il le refusait».

Pour compléter ce tableau, il convient de dire un mot aussi sur les qualités idéales d'un bon maître enseignant. Celui-ci doit être d'âge mûr, parfait dans son art et connaissant les différentes méthodes d'enseignement de l'art musical. Il doit posséder le savoir théorique, être chevronné dans la pratique, doté d'une voix claire et belle, d'une puissance équilibrée, qui ne s'étrangle pas et qui n'est pas désagréable à entendre. Ces conditions sont d'autant plus importantes qu'il s'agit d'un enseignement oral et non systématisé qui, dans une large mesure, rend l'élève dépendant de son maître. Celui-ci, en effet, devient pour lui le principal modèle à imiter jusqu'au stade où son imagination créatrice lui permet de s'en détacher. Les étapes de cette démarche complexe peuvent se résumer ainsi: acquisition des connaissances de base et développement des qualités vocales, familiarisation avec le répertoire, mémorisation d'un grand nombre de chants de genres différents en s'exerçant à imiter leur modèle, et enfin, si le talent du postulant le permet, la mise en valeur de ses apports personnels.

\section{L'excellence du chanteur-musicien}

A la lumière de ce que nous venons d'exposer en détail, nous nous proposons de brosser, en guise de sommaire, l'image idéale du parfait chanteur-musicien. La question de savoir quelles sont les conditions qui font un bon chanteur-musicien

19 Ziryāb, musicien bagdadien et élève du célèbre Isḥāq al-Mawșilī (m. 850), se voit repoussé par la jalousie de son maître; il s'embarque, en 821, pour l'Andalousie sur l'invitation du calife 'Abd al-Raḥmān. A Cordoue, il devient le musicien en chef de la cour et le fondateur de l'école musicale andalouse. 
est souvent posée dans les sources; elle fait son apparition déjà au moment où le nouvel art commence à se cristalliser après l'avènement de l'Islam.

En synthétisant les diverses opinions, nous pouvons retenir les conditions suivantes: en dehors de dispositions musicales innées, de qualités vocales recherchées et de vertus artistiques admises et normalisées, le parfait chanteur-musicien devait se distinguer par sa vaste culture, son éloquence, son art de discourir avec compétence sur les divers domaines du savoir. Répondant à cet idéal, le parfait chanteur-musicien des premiers siècles de l'Islam arrivait à manier avec bonheur aussi bien la musique que la poésie, et grâce à ses compositions, les portes de la cour s'ouvraient au poète dont les vers avaient été mis en musique.

Un musicien qui, de l'avis de tous ses contemporains, remplissait à la perfection ces conditions, fut le célèbre Ishạa al-Mawșilī (m. 850). Son éducation avait été assurée par les meilleurs professeurs de lecture coranique, de grammaire, de poésie, de la science de la tradition (hadith) et de jurisprudence. Ses connaissances lui permettaient aussi bien de tenir sa place dans une assemblée savante que de mener une conversation distrayante. Ses trente-neuf écrits sur la musique couronnent ses exploits exceptionnels; mais hélas! ils sont tous perdus $^{20}$. Il y a là de quoi faire rêver tout musicien de valeur, quels que soient le temps et la culture auxquels il appartient.

Parmi les définitions de l'excellence du musicien qui abondent dans la littérature, nous trouvons les qualités suivantes: «Avoir de la vivacité d'esprit et de la subtilité dans l'assimilation; bien contrôler la respiration; savoir manipuler les différents genres musicaux; être capable de deviner la pensée et les souhaits de ses auditeurs en donnant satisfaction au désir de chacun tout en se mettant d'accord avec le sentiment qu'il éprouve».

Comme complément à ces idées, glanées dans les propos des grands maîtres du passé, nous aimerions citer en guise de conclusion une définition d'un grand musicien du premier siècle de l'Islam réunissant de façon condensée les aspects essentiels discutés dans cette étude.

Répondant à une question sur les qualités qui distinguent le parfait chanteur-musicien, question formulée par Mālik ibn al-Samah (m. env. 136/754), Ibn Suryadj (m. 107/726) dit: «[C'est] le musicien qui enrichit les mélodies, qui a un souffle long, qui confère de la proportion aux mesures, qui donne de l'emphase à la prononciation, qui respecte les inflexions grammaticales, qui soutient les notes longues pendant toute leur durée, qui détache distinctement les notes brèves et qui, enfin, utilise correctement les différents genres rythmiques ». Quand cette définition fut portée à la connaissance du musicien Ma'bad (m. 125/743), il s'exclama: «S'il y avait un Coran pour la musique, il ne saurait pas être autrement».

20 La liste complète de ses écrits figure dans le catalogue des livres en langue arabe Kitāb al-fihrist écrit par ibn al-Nadīm (m. 995). 


\section{Bibliographie}

ABBAS Ihsan (ed.)

1968 Al-Maqqāiri: Naf̣̣ al-țīb min ghuṣn al-andalus al-rațīb... 8 vol. Beirut.

DOZY R., DUGAT G., KREHL Ludolf et WRIGHT W.

1855-1861 Analectes sur l'histoire et la littérature des Arabes d'Espagne, par al-Maqqārī. Leiden: Brill.

\section{DURING Jean}

1988 Musique et extase. L'audition spirituelle dans la tradition soufie. Paris: Albin Michel.

ERLANGER baron Rodolphe d'

1930-1959 La musique arabe. 6 vol. Paris: Geuthner.

FARMER Henry-George

1929 A history of Arabian music to the XIIIth century. London: Luzac.

GHATTAS A. et HEFNI M. (ed.)

1967 Kitāb al-mūsīqī al-kabìr d'al-fārābì. Cairo.

MASSIGNON Louis

1958-1961 «Voyelles sémitiques et sémantique musicale», in: Encyclopédie de la musique I: 79-83. Paris: Fasquelle.

MOLÉ Marijan

1963 «La danse extatique en Islam», in: Les danses sacrées. Paris: Seuil. [Sources orientales 6] : pp. $145-280$.

RITTER Hellmuth

1955 Das Meer der Seele. Leiden: Brill.

ROBSON James (ed. and trad.)

1938 Tracts on Listening to Music. Being dhamm al-malāhī by Ibn abi'l Dunya and Bawāriq alilmā' by Majd al-dìn al-Ghazālī. London.

ROSEN Haiim

1989 «Nouveaux regards sur l'expression poétique d'Homère», Revue des études grecques (Paris) CII, 2: 263-83.

ROSENTHAL Franz (trad.)

1958 The Muqaddimah. An Introduction to History. 3 vol. New York: Bollingen.

ROUANET Jules

1922 «La musique arabe», in: A. Lavignac Encyclopédie de la musique et dictionnaire du Conservatoire. $1^{\text {re }}$ partie, t. V: 2676-2812. Paris: Delagrave.

SICARD R.

1987 «L'amour dans la rișālat al-qiyān - Essai sur les esclaves-chanteuses - de Gāḥiz », Arabica XXXIV : 326-38.

SHILOAH Amnon

1972 La perfection des connaissances musicales. Paris: Geuthner.

1979 The Theory of Music in Arabic Writings. München: Répertoire international de sources musicales.

SLANE W.M.G., baron de

1863-1868 Les prolégomènes d'Ibn Khaldun... Reproduction photomécanique de la première partie des tomes XIX, XX et XXI des notices et extraits des mss. de la BN, Paris.

TALBI M.

1958 «La Qirā’ah bi-l-alḥān», Arabica V: 183-90.G 